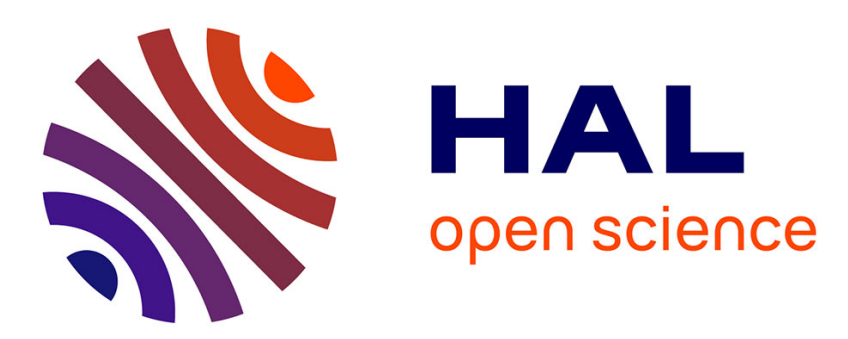

\title{
Croisements interspécifiques entre quelques Brassicae et Brassica napus L. Analyse génomique des hybrides et perspectives d'obtention de systèmes d'androstérilité chez le colza
}

Patrick Rousselle, Frédérique Eber

\section{To cite this version:}

Patrick Rousselle, Frédérique Eber. Croisements interspécifiques entre quelques Brassicae et Brassica napus L. Analyse génomique des hybrides et perspectives d'obtention de systèmes d'androstérilité chez le colza. Agronomie, 1983, 3 (2), pp.153-159. hal-00884494

\author{
HAL Id: hal-00884494 \\ https://hal.science/hal-00884494
}

Submitted on 1 Jan 1983

HAL is a multi-disciplinary open access archive for the deposit and dissemination of scientific research documents, whether they are published or not. The documents may come from teaching and research institutions in France or abroad, or from public or private research centers.
L'archive ouverte pluridisciplinaire $\mathbf{H A L}$, est destinée au dépôt et à la diffusion de documents scientifiques de niveau recherche, publiés ou non, émanant des établissements d'enseignement et de recherche français ou étrangers, des laboratoires publics ou privés. 


\section{Croisements interspécifiques entre quelques Brassicae et Brassica napus L. Analyse génomique des hybrides et perspectives d'obtention de systèmes d'androstérilité chez le colza}

Patrick ROUSSELLE \& Frédérique EBER

I.N.R.A., Station d'Amélioration des Plantes, Centre de Recherches de Rennes, B.P. 29, F-35650 Le Rheu

\section{RÉSUMÉ}

Stérilité mâle, Cytogénétique, Culture d'embryon in vitro,

Fertilité pollinique.
Pour induire de nouvelles sources d'androstérilité cytoplasmique chez le colza, la création de géniteurs alloplasmiques est une des voies à privilégier. Pour ce faire, nous avons effectué quatre séries de croisements interspécifiques :

Brassica nigra Koch. $\times$ B. napus L., B. campestris L. $\times$ B. napus, B. carinata A. Br. $\times$ B. napus, B. juncea Coss. $\times$ B. napus.

Nous avons réalisé la culture in vitro des embryons immatures issus de ces croisements pour obtenir un nombre suffisant de plantes. Les analyses cytogénétiques de ces hybrides interspécifiques permettent de confirmer les hypothèses connues d'homéologie entre les génomes. Dans certains cas, nous avons noté la présence d'un nombre supérieur de bivalents ou multivalents par rapport à la méiose théorique ; ceci peut favoriser les recombinaisons entre génomes différents.

Les analyses de fertilité des plantes en $2^{\mathrm{e}}$ génération montrent que les croisements dans lesquels $B$. campestris est utilisé ont peu de chance de déboucher sur une stérilité mâle cytoplasmique. Par contre les 3 autres croisements présentent une très grande variabilité de la fertilité pollinique.

La sélection envisagée a pour objectif l'obtention d'un système d'androstérilité cytoplasmique (plantes mâle stériles et restaurateurs) tout en évitant les cas de stérilité mâle dus à des aberrations chromosomiques.

\section{SUMMARY}

Male sterility,

Cytogenetics,

Embryo culture,

Pollen fertility.
Interspecific crosses between some Brassica species and Brassica napus L. Genome analysis of the hybrids and possibilities of obtaining male sterility in rape.

The production of alloplasmic lines is an excellent procedure for the development of additional sources of cytoplasmic male sterility in rape (Brassica napus L.). For this purpose four series of interspecific crosses were made, between Brassica nigra Koch., B. campestris L., B. carinata A.Br. and B. juncea Coss. respectively and B. napus $\mathrm{L}$.

Interspecific embryos were cultured in vitro in order to obtain enough plants. Results from the cytogenetic analysis of these hybrids confirmed the existing hypothesis about homeology between genomes within the genus Brassica. In some cases, more bivalents or multivalents than expected were observed, which may improve recombination among the different genomes.

The fertility of the plants in the second generation was analysed. Crosses involving $B$. campestris were not likely to result in male-sterile plants. However, the three other crosses showed a wide variability for pollen fertility.

Our future breeding programme has as its main objective a cytoplasmic male sterility system (male sterile and restorer plants) from which chromosomal aberrations are excluded.

\section{INTRODUCTION}

L'utilisation des croisements interspécifiques a principalement servi à introduire un ou plusieurs caractères présents chez une espèce voisine; la résistance aux parasites est l'exemple le plus courant. Peu d'intérêt était porté aux plantes mâle stériles obtenues, en particulier chez les espèces autogames. Dans la descendance de croisements entre Aegilops caudata L. et Triticum aestivum (L.) Thell., KIHARA (1951) observa des plantes mâles stériles à déterminisme génocytoplasmique et souligna leur importance pour la création d'hybrides. Le croisement entre 2 espèces différentes apparaît comme un moyen d'obtention de tels systèmes d'androstérilité. EDWARDSON (1970) a répertorié 
32 cas pour lesquels un croisement interspécifique a conduit à l'obtention de plantes androstériles, notamment chez le tournesol et chez le blé :

- LeClerCQ $(1969,1971)$ a mis en évidence l'intérêt de Helianthus petiolaris Nutt. pour la création de lignées androstériles cytoplasmiques chez le tournesol (Helianthus annuus L.). Ces lignées ont rendu possible la production de semences hybrides aux USA et en Europe.

- Un grand nombre d'espèces apparentées au blé ont été analysées pour leur aptitude à la création de systèmes d'androstérilité cytoplasmique (TSUNEWAKI, 1978). WILSON \& Ross (1962) ont obtenu des géniteurs utilisables à partir du croisement entre Triticum timopheevi Zhuk. et $T$. aestivum (L.) Thell.. La fertilité incomplète des hybrides restaurés (AURIAU et al., 1973) semble pouvoir être améliorée par l'utilisation de nouveaux géniteurs (AURIAU et al., 1979).

Chez le colza (Brassica napus L.), la valorisation de la vigueur hybride nécessite un strict contrôle de la pollinisation qui peut être obtenu par l'androstérilité cytoplasmique. Deux sont d'origine intraspécifique (SHIGA, 1976 ; THOMPSON, 1972). Deux proviennent d'espèces voisines du colza mais diffèrent quant à leur nature. La stérilité mâle intraspécifique du radis (OGURA, 1968) a été transférée chez le chou (BANNERot et al., 1974) puis chez le colza où un certain nombre d'inconvénients ne permettent pas actuellement son utilisation (Rousselle, 1981). A partir du croisement entre Brassica nigra Koch. et B. oleracea L., PEARSON (1972) a obtenu directement un système d'androstérilité cytoplasmique utilisé chez le broccoli (DICKSON, 1975). L'introgression de ce système chez le colza est en cours.

Du fait des inconvénients liés aux systèmes d'androstérilité cytoplasmique déjà existants chez le colza, il nous paraît indispensable de multiplier les origines de cytoplasmes. L'exemple du blé et du tournesol montre l'intérêt de l'obtention de plantes androstériles par croisements interspécifiques mais aussi les difficultés liées à la restauration de la fertilité.

L'étude dont nous présentons ici les observations faites sur les 2 premières générations a pour objectif principal l'obtention de lignées alloplasmiques de colza androstériles et restauratrices. Il nous est apparu indispensable de contrôler la composition génomique des hybrides obtenus et de comparer nos résultats avec les hypothèses d'homéologie connues entre les espèces du genre Brassica utilisées. Nous avons réalisé l'analyse de la fertilité des plantes de $2^{\text {c }}$ génération, uniquement dans le cas où $B$. napus était utilisé comme parent mâle.

\section{MATÉRIEL ET MÉTHODES}

\section{A. Matériel végétal}

Nous n'avons utilisé qu'une seule lignée de $B$. napus: «Crésor», cultivar de printemps. Deux cultivars de navette (B. campestris L.) ont été employés : «Daisy » et « Chicon». La Station d'Amélioration des Plantes (INRA) de Dijon nous a fourni la moutarde noire ( $B$. nigra Koch.). La moutarde d'Abyssinie ( $B$. carinata A. Br.) provient d'Ethiopie : cultivar "Awassa». Deux moutardes brunes (B. juncea Coss.) ont été utilisées, l'une est le cultivar «Brunet » et l'autre provient de Gatersleben. L'ensemble de ces espèces, avec le chou ( $B$. oleracea L.), forme le triangle de U (1935) (fig. 1). Le chou, qui apparaît comme

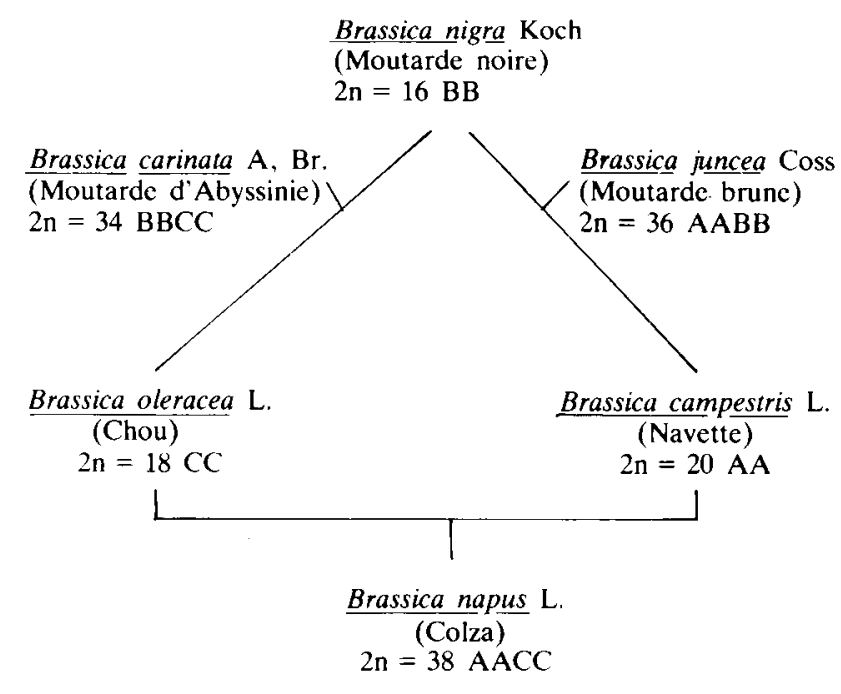

Figure 1

Relations génomiques entre quelques Brassicae alliés au colza (U, 1935).

Genome relationships within Brassica species related to rape (U, 1935).

étant le parent maternel du colza, n'a pas été utilisé en raison d'un manque de concordance de floraison.

\section{B. Plan de croisement}

Les croisements suivants ont été réalisés dans les deux sens :

\section{$B$. campestris $\times B$. napus \\ $B$. nigra $\times B$. napus \\ $B$. carinata $\times B$. napus \\ $B$. juncea $\times B$. napus}

Les plantes $\mathrm{F} 1$ ont été laissées en libre pollinisation à l'intérieur de chaque type de croisement pour obtenir la $2^{c}$ génération $(\mathrm{G} 2)$.

\section{Culture in vitro d'embryons immatures}

Les plantes mères sont cultivées en serre. Environ 10 à $15 \mathrm{j}$ après la pollinisation manuelle, la jeune silique est prélevée et désinfectée par simple immersion dans l'alcool absolu. Après dissection de l'ovule, l'embryon issu de croisement interspécifique est prélevé et déposé sur le milieu de MonNier (1974) dans une boîte de Petri.

La teneur en saccharose de ce milieu varie de $120 \mathrm{~g} .1^{-1}$ pour des embryons globulaires à $50 \mathrm{~g} .1^{-1}$ pour des embryons cordiformes. La boîte de Petri est ensuite placée dans une enceinte climatisée : $16 \mathrm{~h}$ d'éclairement à 10000 lux et à $25^{\circ} \mathrm{C}$ suivies de $8 \mathrm{~h}$ d'obscurité à $20^{\circ} \mathrm{C}$ pendant 8 à $15 \mathrm{j}$ suivant le développement de l'embryon. Lorsque ce dernier a atteint environ $1 \mathrm{~cm}$, il est repiqué dans un tube de culture contenant le même milieu mais avec une teneur en saccharose plus faible ( 20 à $25 \mathrm{~g} . \mathrm{l}^{-1}$ ); le tube de culture est ensuite replacé dans la même enceinte climatisée. Lorsque la plantule est suffisamment développée, elle est repiquée en mini-serre sur de la vermiculite arrosée d'une solution nutritive contenant les macro-éléments de MONNIER (1974) 2 fois moins concentrés. Cette mini-serre est placée dans la même enceinte. Lorsque le développement est jugé satisfaisant, les plantes sont rempotées en serre avant le repiquage définitif au champ. 


\section{Observations cytogénétiques et viabilité pollinique}

Les observations des méioses polliniques en métaphase I des cellules mères du pollen sont réalisées selon la technique utilisée chez le blé (DosBA \& DoussinaulT, 1978).

La fertilité pollinique est déterminée à partir du pourcentage de grains de pollen colorés au carmin acétique de Belling. Nous appelons If ce pourcentage, Is $(=100-$ If $)$ étant l'indice de stérilité pollinique. Pour chaque plante, 4 fleurs sont prélevées et, pour chaque fleur, 500 grains au moins sont comptés pour les 2 classes (fertile et stérile).

\section{RÉSULTATS ET DISCUSSION}

\section{A. Obtention de plantes hybrides}

Toutes les combinaisons ont été obtenues précédemment pour étudier les relations génomiques chez les Brassicae (MORICE, 1963), la plupart sans culture in vitro des embryons immatures. Cette dernière permet d'améliorer le rendement. Le nombre d'embryons obtenus est très important dans les croisements faisant intervenir $B$. campestris, une des espèces parentales du colza (fig. 1). Par contre, il est très faible lorsque $B$. nigra est utilisé ; cette espèce n'a pas de génome commun avec le colza. Les 2 autres espèces ( $B$. carinata et $B$. juncea) qui sont des amphiploïdes ont un génome commun avec le colza. Le nombre d'embryons prélevés dans les croisements entre ces 2 espèces et le colza est intermédiaire (tabl. 1). Ainsi, il apparaît dans ces croisements que le taux de réussite des croisements est proportionnel au degré de parenté entre les génomes.

Le nombre de plantes hybrides survivantes au stade floraison représente 27 p. 100 du nombre total d'embryons prélevés, tous croisements confondus. Les embryons qui n'ont pas survécu (63 p. 100) ont, sans doute, mal supporté soit les teneurs en sucre trop élevées soit le repiquage en mini-serre. La morphologie des hybrides est intermédiaire entre celles des 2 parents pour les caractères foliaires et floraux. Dans la plupart des cas, le pollen, bien que très abondant, a une fertilité faible mais suffisante pour obtenir la génération suivante. Quatre plantes du croisement $B$. juncea $\times B$. napus présentent des étamines complètement atrophiées. Deux d'entre elles ont eu une descendance.

\section{B. Comportement méiotique des hybrides interspécifi- ques}

Les relations génomiques entre les espèces utilisées dans nos croisements sont bien connues et schématisées dans le triangle de U (1935) (fig. 1). Pour chaque croisement, nous pouvons donc prévoir la configuration théorique des méioses. Avant de décrire ces hybrides, précisons que les 5 espèces parentales ont été contrôlées et que leurs méioses ne présentaient que des bivalents avec une proportion variable de droits et d'anneaux.

\section{B. campestris $\times \mathrm{B}$. napus et réciproques}

La navette (B. campestris) et le colza (B. napus) (fig. 1) ont en commun le génome $\mathrm{A}(\mathrm{n}=10)$, le génome $\mathrm{C}(\mathrm{n}=9)$ provenant du chou ( $B$. oleracea). Le comportement méiotique attendu est donc: 9 univalents et 10 bivalents. Le tableau 2 indique le comportement moyen de chacun des hybrides obtenus. L'analyse du $\chi^{2}$ d'homogénéité montre qu'il n'y a pas de différence significative entre les plantes, quel que soit le sens du croisement : $\chi^{2}=4,21$; $0,75 \leqslant P \leqslant 0,90$. De plus, la moyenne de ces croisements (9,77 univalents et 9,62 bivalents) ne s'écarte pas de la méiose théorique $\left(\chi^{2}=0,08 ; 0,75 \leqslant P \leqslant 0,90\right)$. Ces résultats sont en accord avec les observations généralement faites

TABLEAU 1

Obtention des hybrides.

Hybrids produced.

\begin{tabular}{|c|c|c|c|}
\hline Croisements réalisés & $\begin{array}{l}\text { Nombre de } \\
\text { fleurs } \\
\text { pollinisées }\end{array}$ & $\begin{array}{l}\text { Nombre } \\
\text { d'embryons } \\
\text { prélevés }\end{array}$ & $\begin{array}{c}\text { Nombre de plantes } \\
\text { obtenues en } \\
\text { floraison }\end{array}$ \\
\hline $\begin{array}{l}\text { B. napus }\left({ }^{1}\right) \times B . \text { carinata }\left({ }^{2}\right) \\
\text { B. carinata } \times B . \text { napus }\end{array}$ & $\begin{array}{r}165 \\
84\end{array}$ & $\begin{array}{l}50 \\
17\end{array}$ & $\begin{array}{l}3 \\
2\end{array}$ \\
\hline $\begin{array}{l}\text { B. napus }\left({ }^{1}\right) \times B . \text { campestris }\left({ }^{3}\right) \\
\text { B. campestris } \times \text { B. napus }\end{array}$ & $\begin{array}{l}90 \\
90\end{array}$ & $\begin{array}{l}46 \\
28\end{array}$ & $\begin{array}{l}7 \\
3\end{array}$ \\
\hline $\begin{array}{l}\text { B. napus }\left({ }^{1}\right) \times \text { B. campestris }\left({ }^{4}\right) \\
\text { B. campestris } \times \text { B. napus }\end{array}$ & $\begin{array}{l}75 \\
36\end{array}$ & $\begin{array}{l}65 \\
30\end{array}$ & $\begin{array}{l}13 \\
11\end{array}$ \\
\hline $\begin{array}{l}\text { B. napus }\left({ }^{1}\right) \times B . \text { nigra }\left({ }^{5}\right) \\
\text { B. nigra } \times \text { B. napus }\end{array}$ & $\begin{array}{r}120 \\
54\end{array}$ & $\begin{array}{r}1 \\
14\end{array}$ & $\begin{array}{l}1 \\
3\end{array}$ \\
\hline $\begin{array}{l}\text { B. napus }\left({ }^{1}\right) \times B . \text { juncea }\left({ }^{6}\right) \\
\text { B. juncea } \times \text { B. napus }\end{array}$ & $\begin{array}{l}45 \\
24\end{array}$ & $\begin{array}{r}8 \\
51\end{array}$ & $\begin{array}{r}2 \\
17\end{array}$ \\
\hline $\begin{array}{l}\text { B. napus }\left({ }^{1}\right) \times \text { B. juncea }\left({ }^{7}\right) \\
\text { B. juncea } \times \text { B. napus }\end{array}$ & $\begin{array}{l}45 \\
30\end{array}$ & $\begin{array}{r}7 \\
13\end{array}$ & $\begin{array}{l}6 \\
1\end{array}$ \\
\hline $\begin{array}{l}\text { (1) Cultivar «Crésor» } \\
\left({ }^{2}\right) \text { Cultivar «Awassa » } \\
\left({ }^{3}\right) \text { Cultivar "Daisy " } \\
\left({ }^{4}\right) \text { Cultivar "Chicon " }\end{array}$ & $\begin{array}{l}\text { (5) Cultivar « I.N.R.A. Dijon } 3279 \text { " } \\
\text { (6) Cultivar « Gatersleben Bra } 427-72 \text { » } \\
\text { (7) Cultivar « Brunet » }\end{array}$ & & \\
\hline
\end{tabular}


TABLEAU 2

Comportement méiotique des hybrides réciproques entre Brassica campestris $L$. et Brassica napus $L$. Meiotic behaviour of reciprocal hybrids between Brassica campestris $L$. and Brassica napus $L$.

\begin{tabular}{|c|c|c|c|c|c|c|c|c|c|}
\hline & \multirow{2}{*}{$\begin{array}{c}\mathrm{N}^{\circ} \\
\text { plante }\end{array}$} & \multirow{2}{*}{$\underset{\ell}{\mathrm{nb}}$} & \multirow{2}{*}{$2 n$} & \multicolumn{3}{|c|}{ Univalents } & \multicolumn{3}{|c|}{ Bivalents } \\
\hline & & & & $\bar{X}$ & extr. & mode & $\dot{\mathrm{X}}$ & extr. & mode \\
\hline \multirow{4}{*}{$\begin{array}{c}\text { B. napus } \\
\times \\
\text { B. campestris }\end{array}$} & 559 & 50 & 29 & 9,96 & $9-13$ & 9 & 9,52 & $8-10$ & 10 \\
\hline & 561 & 55 & 29 & 9,36 & $7-11$ & 9 & 9,82 & 9.11 & 10 \\
\hline & 579 & 13 & 29 & 9,31 & $9-11$ & 9 & 9,85 & $9-10$ & 10 \\
\hline & 583 & 52 & 29 & 10,12 & $9-13$. & 9 & 9,44 & $8-10$ & 10 \\
\hline \multirow{4}{*}{$\begin{array}{c}\text { B. campestris } \\
\times \\
\text { B. napus }\end{array}$} & 411 & 50 & 29 & 9,80 & $9-13$ & 9 & 9,60 & $3-10$ & 10 \\
\hline & 418 & 27 & 29 & 9,37 & $9-13$ & 9 & 9,81 & $8-10$ & 10 \\
\hline & 420 & 24 & 29 & 9,42 & $9-11$ & 9 & 9,79 & $9-10$ & 10 \\
\hline & 429 & 60 & 29 & 10,07 & $9-15$ & 9 & 9,47 & $7-10$ & 10 \\
\hline
\end{tabular}

TABLEAU 3

Comportement méiotique des hybrides réciproques entre Brassica carinata $A$. Br. et Brassica napus $L$. Meiotic behaviour of reciprocal hybrids between Brassica carinata $A$. Br. and Brassica napus $L$.

\begin{tabular}{|c|c|c|c|c|c|c|c|c|c|c|c|c|c|c|c|}
\hline & \multirow{2}{*}{$\begin{array}{c}n^{\circ} \\
\text { Plante }\end{array}$} & \multirow{2}{*}{$\stackrel{\mathrm{nb}}{C}$} & \multirow{2}{*}{$2 n$} & \multicolumn{3}{|c|}{ Univalents } & \multicolumn{3}{|c|}{ Bivalents } & \multicolumn{3}{|c|}{ Trivalents } & \multicolumn{3}{|c|}{ Quadrivalents } \\
\hline & & & & $\ddot{\mathbf{X}}$ & extr. & mode & $\overline{\mathrm{X}}$ & extr. & mode & $\bar{X}$ & extr. & mode & $\overline{\mathrm{X}}$ & extr. & mode \\
\hline $\begin{array}{c}\text { B. napus } \\
\times \\
\text { B. carinata }\end{array}$ & $\begin{array}{l}43 \\
86\end{array}$ & $\begin{array}{r}20 \\
8\end{array}$ & $\begin{array}{l}36 \\
36\end{array}$ & $\begin{array}{l}16,35 \\
13,63\end{array}$ & $\begin{array}{r}15-20 \\
6-20\end{array}$ & $\begin{array}{l}16 \\
16\end{array}$ & $\begin{array}{r}9,45 \\
10,75\end{array}$ & $\begin{array}{l}7-10 \\
8-15\end{array}$ & $\begin{array}{l}10 \\
10\end{array}$ & $\begin{array}{l}0,05 \\
0,13\end{array}$ & $\begin{array}{l}0-1 \\
0-1\end{array}$ & $\begin{array}{l}0 \\
0\end{array}$ & $\begin{array}{l}0,15 \\
0,13\end{array}$ & $\begin{array}{l}0-1 \\
0-1\end{array}$ & $\begin{array}{l}0 \\
0\end{array}$ \\
\hline $\begin{array}{c}\text { B. carinata } \\
\times \\
\text { B. napus }\end{array}$ & $\begin{array}{l}400 \\
401\end{array}$ & $\begin{array}{l}52 \\
22\end{array}$ & $\begin{array}{l}36 \\
36\end{array}$ & $\begin{array}{l}15,94 \\
16,54\end{array}$ & $\begin{array}{r}8-18 \\
16-18\end{array}$ & $\begin{array}{l}16 \\
16\end{array}$ & $\begin{array}{r}10,00 \\
9,73\end{array}$ & $\begin{array}{l}9-14 \\
9-10\end{array}$ & $\begin{array}{l}10 \\
10\end{array}$ & $\begin{array}{c}0,02 \\
-\end{array}$ & $\begin{array}{l}0-1 \\
-\end{array}$ & $\begin{array}{l}0 \\
-\end{array}$ & - & - & - \\
\hline
\end{tabular}

sur ce type de croisements et formulées en particulier par MORINAGA (1929).

\section{B. carinata $\times$ B. napus et réciproque (tabl. 3)}

Ces deux espèces ont en commun le génome $\mathrm{C}(\mathrm{n}=9)$; la $1^{\text {re }}$ possède de plus le génome $B(n=8)$. La méiose attendue d'un tel hybride est donc composée de 18 univalents et de 9 bivalents. Le calcul du $\chi^{2}$ montre une certaine hétérogénéité entre les plantes $n^{\circ} 43$ et $n^{\circ} 86 \quad\left(\chi^{2}=4,18\right.$; $0,25 \geqslant \mathrm{P}^{\prime} \geqslant 0,10$ ) et une bonne homogénéité entre les plantes $n^{\circ} 43,400$ et $401\left(\chi^{2}=0,88 ; 0,90 \geqslant P \geqslant 0,75\right)$. Le faible nombre de cellules observées dans la plante $n^{\circ} 86$ peut être la cause de cette hétérogénéité apparente de la méiose. La comparaison entre chacune des méioses observées et la méiose théorique montre un déficit d'univalents au profit des chromosomes appariés (bivalents et multivalents). U (1935) avait également observé, dans ce type de croisement, un nombre important de trivalents. Ainsi, le taux d'appariement total observé est-il largement supérieur à celui attendu et permet-il de supposer un appariement entre chromosomes de génomes différents. Ce type d'appariements avait été noté dans les croisements faisant intervenir $B$. carinata et $B$. campestris, réalisés pour obtenir des plantes possédant les 3 génomes à l'état monoploïde (MorinaGA, 1931 ; Howard, 1942 ; MizUSHIMA, 1944, 1950 ; IWASA, 1951).

\section{B. juncea $\times$ B. napus (tabl. 4)}

Le génome $\mathrm{A}(\mathrm{n}=10)$ est commun à ces deux espèces; $B$. juncea apporte en plus le génome $\mathrm{B}(\mathrm{n}=8)$. Ainsi la méiose théorique de l'hybride étudié est-elle composée de 17 univalents et 10 bivalents. Le comportement méiotique de l'ensemble de ces plantes n'est pas très homogène puisque, dans certains cas, on observe un nombre non négligeable de trivalents et de quadrivalents. Dans l'ensemble, nous avons noté peu de déviation par rapport à la méiose théorique (SinSKAIA, 1927 ; HEYN, 1977; RoY, 1978).

\section{Fertilité pollinique des G2}

Le but principal de cette étude réside dans l'obtention d'un système d'androstérilité cytoplasmique chez le colza que nous recherchons dans une association entre le noyau de $B$. napus et le cytoplasme d'une espèce différente (lignée alloplasmique). Pour cette raison, nous avons limité notre étude de la fertilité pollinique aux croisements de ce type sans examiner les croisements réciproques.

\section{B. nigra $\times$ B. napus}

Une seule plante $F 1$ a donné 4 plantes en G2. Deux d'entre elles présentent une forte stérilité (Is $>75 \mathrm{p} .100$ ) et 1 plante, une bonne fertilité (Is $=14,4$ p. 100). La $4^{\mathrm{e}}$ n'a pu être analysée.

\section{B. campestris $\times$ B. napus (tabl. 5)}

Toutes les plantes proviennent de croisements utilisant le cultivar «Chicon». Dans tous les cas, nous avons noté un développement normal des anthères, aucune n'est atrophiée ou absente. L'ensemble du matériel apparaît comme ayant une bonne fertilité (Is moyen : 24,7 p. 100 , Is $\leqslant 20$ p. 100 
TABLEAU 4

Comportement méiotique des hybrides Brassica juncea Coss. $\times$ Brassica napus $L$.

Meiotic behaviour of hybrids of the cross Brassica juncea Coss. $\times$ Brassica napus $L$.

\begin{tabular}{|c|c|c|c|c|c|c|c|c|c|c|c|c|c|c|c|}
\hline & \multirow{2}{*}{$\begin{array}{c}\mathbf{N}^{\circ} \\
\text { plante }\end{array}$} & \multirow{2}{*}{$\begin{array}{c}\mathrm{nb} \\
\ell\end{array}$} & \multirow{2}{*}{$2 n$} & \multicolumn{3}{|c|}{ Univalents } & \multicolumn{3}{|c|}{ Bivalents } & \multicolumn{3}{|c|}{ Trivalents } & \multicolumn{3}{|c|}{ Quadrivalents } \\
\hline & & & & $\overline{\mathrm{X}}$ & extr. & mode & $\bar{X}$ & extr. & mode & $\overline{\mathbf{X}}$ & extr. & mode & $\overline{\mathrm{X}}$ & extr. & mode \\
\hline \multirow{8}{*}{$\begin{array}{l}\text { B. juncea } \\
\times \\
\text { B. napus }\end{array}$} & 225 & 55 & 37 & 17,07 & $15-19$ & 17 & 9,96 & $9-11$ & 10 & - & - & - & 一 & - & - \\
\hline & 227 & 89 & 37 & 17,00 & $15-19$ & 17 & 9,96 & $8-11$ & 10 & 0,02 & 0.1 & 0 & - & - & - \\
\hline & 228 & 56 & 37 & 17,04 & $15-19$ & 17 & 9,88 & $9-11$ & 10 & 0,07 & $0-1$ & 0 & - & - & - \\
\hline & 258 & 40 & 37 & 16,85 & $15-19$ & 17 & 10,00 & $8-11$ & 10 & 0,05 & $0-1$ & 0 & - & - & - \\
\hline & 271 & 70 & 37 & 16,93 & $15-19$ & 17 & 9,77 & $6-11$ & 10 & 0,16 & 0.2 & 0 & 0,01 & $0-1$ & 0 \\
\hline & 272 & 62 & 37 & 16,93 & $15-19$ & 17 & 10,03 & $9-11$ & 10 & - & - & - & - & - & - \\
\hline & 286 & 14 & 37 & 17,28 & $15-19$ & 17 & 9,86 & $9-11$ & 10 & - & - & - & - & - & - \\
\hline & 288 & 57 & 37 & 16,79 & $15-18$ & 17 & 10,05 & 8-11 & 10 & 0,04 & $0-1$ & 0 & - & - & - \\
\hline
\end{tabular}

TABLEAU 5

Stérilité pollinique de la $G 2$ du croisement entre Brassica campestris $L$. et Brassica napus $L$.

Pollen sterility in the $G 2$ of the cross

Brassica campestris $L . \times$ Brassica napus $L$.

\begin{tabular}{|c|c|c|c|}
\hline \multirow{2}{*}{ Code plante $F_{1}$} & \multicolumn{3}{|r|}{ Plantes G2 } \\
\hline & Code & Is & Observations \\
\hline 429 & $\begin{array}{l}\mathrm{A} \\
\mathrm{B} \\
\mathrm{C} \\
\mathrm{D} \\
\mathrm{E} \\
\mathrm{F} \\
\mathrm{GH}\end{array}$ & $\begin{array}{l}14,9 \\
12,2 \\
36,1 \\
12,3 \\
37,1\end{array}$ & $\begin{array}{l}\text { Grains à moitié colorés }\left({ }^{1}\right) \\
\text { Pollen présent non dénombré }\left({ }^{2}\right)\end{array}$ \\
\hline 420 & $\begin{array}{l}\text { A } \\
\text { B } \\
\text { C } \\
\text { D } \\
\text { E } \\
\text { F } \\
\text { G }\end{array}$ & $\begin{array}{l}35,4 \\
28,6 \\
16,4 \\
53,1 \\
34,8 \\
15,8 \\
11,9\end{array}$ & \\
\hline 425 & $\begin{array}{l}\text { A } \\
\text { B }\end{array}$ & $\begin{array}{r}3,2 \\
41,6\end{array}$ & \\
\hline 416 & $\begin{array}{l}\text { A } \\
\text { B } \\
C \\
D \\
E \\
F \\
\text { G } \\
\text { HIJ }\end{array}$ & $\begin{array}{r}23,9 \\
5,2 \\
77,7 \\
77,5 \\
13,9 \\
15,6 \\
56,8\end{array}$ & Pollen présent non dénombré $\left({ }^{2}\right)$ \\
\hline $\begin{array}{l}411 \\
421-2 \\
433\end{array}$ & & & $\begin{array}{l}\text { Aucune plante à étamines }\left({ }^{3}\right) \\
\text { atrophiées, pollen présent } \\
\text { non dénombré }\end{array}$ \\
\hline
\end{tabular}

(') Pollen grains half stained.

(2) Pollen grains were not counted.

(3) No plant with aborted stamens.

chez 9 plantes), sauf 2 plantes $n^{\circ} 416 \mathrm{C}$ et $\mathrm{D}$ qui ont une forte stérilité (Is $>75 \mathrm{p} .100$ ). La navette a été souvent utilisée pour augmenter la variabilité génétique du colza et il n'a jamais été mentionné de difficultés dans l'obtention des différentes générations.

SHIGA (1970) mentionne que sur 41 variétés de colza

\section{TABLEAU 6}

Stérilité pollinique de la $G 2$ du croisement entre Brassica carinata $A$. Br. et Brassica napus $L$. Pollen sterility in the $G 2$ of the cross

Brassica carinata $A . B r, \times$ Brassica napus $L$.

\begin{tabular}{|c|c|c|c|}
\hline \multirow{2}{*}{ Code plante $F_{1}$} & \multicolumn{3}{|r|}{ Plantes G2 } \\
\hline & Code & Is & Observations \\
\hline 400 & $\begin{array}{l}\text { A } \\
\text { B } \\
\text { C } \\
\text { D } \\
\text { E } \\
\text { F } \\
\text { G } \\
\text { H } \\
\text { I }\end{array}$ & $\begin{array}{l}85,4 \\
14,3 \\
50,3 \\
48,5 \\
52,2 \\
68,1\end{array}$ & $\begin{array}{l}\text { Pas d'étamine }\left({ }^{1}\right) \\
\text { Pollen présent non dénombré }\left({ }^{2}\right) \\
\text { Pas d'étamine }\left({ }^{1}\right)\end{array}$ \\
\hline 401 & $\begin{array}{l}\text { A } \\
\text { B } \\
\text { C } \\
\text { DEF } \\
\text { GH }\end{array}$ & & $\begin{array}{l}\text { Pas d'étamine }\left({ }^{1}\right) \\
\text { Etamines atrophiées }\left({ }^{3}\right) \\
\text { Etamines atrophiées }\left({ }^{3}\right) \\
\text { Pollen présent non dénombré }\left({ }^{2}\right)\end{array}$ \\
\hline $\begin{array}{l}\text { (1) No stamen. } \\
\text { (2) Pollen grains } \\
\left({ }^{3}\right) \text { Aborted stam }\end{array}$ & $\begin{array}{l}\text { were } r \\
\text { ens. }\end{array}$ & $\mathrm{tco}$ & unted. \\
\hline
\end{tabular}

enregistrées au Japon, 21 au moins ont un ascendant de type $B$. campestris, ce qui corrobore la fertilité élevée des descendances d'un tel croisement.

\section{B. carinata $\times$ B. napus (tabl. 6)}

La G2 de ce croisement présente une grande variabilité et quelques cas extrêmes: 3 plantes n'ont pas d'étamine $\left(\mathrm{n}^{\circ} 400 \mathrm{C}, 400 \mathrm{I}\right.$ et $\left.401 \mathrm{~A}\right) ; 2$ ont des étamines atrophiées $\left(n^{\circ} 401 \mathrm{~B}\right.$ et $\left.401 \mathrm{C}\right)$; une possède un Is fort $\left(\mathrm{n}^{\circ} 400 \mathrm{~A}\right)$; 4 plantes ont un Is voisin de $50\left(\mathrm{n}^{\circ} 400 \mathrm{D}, 400 \mathrm{E}, 400 \mathrm{~F}\right.$ et $400 \mathrm{G}$ ) ; la plante $\mathrm{n}^{\circ} 400 \mathrm{~B}$ est la plus fertile avec Is faible $(14,3$ p. 100).

Cet étalement de la fertilité pollinique doit nous permettre de créer des têtes de familles diversifiées et répondant aux objectifs fixés.

4. B. juncea $\times$ B. napus (fig. 2)

C'est le croisement pour lequel nous avons obtenu le plus grand nombre de plantes en G2. La figure 2 indique, pour 


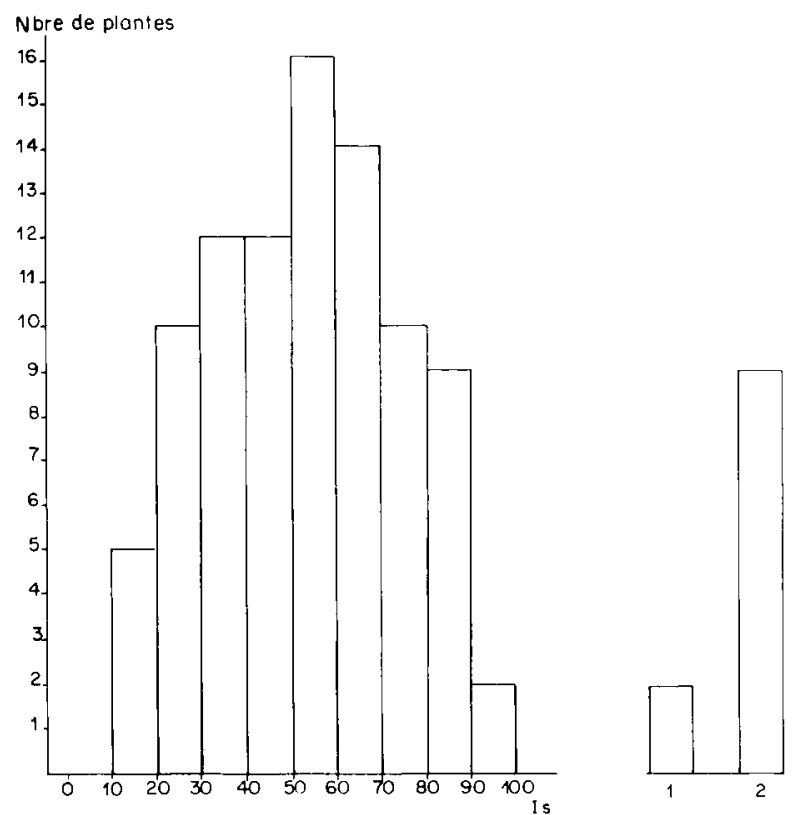

Figure 2

Stérilité pollinique de la $G 2$ du croisement Brassica juncea Coss. $\times$ Brassica napus $L$.

Pollen sterility in the $G 2$ of the cross Brassica juncea Coss. $\times$ Brassica napus $L$.

1 - Plantes à étamines atrophiées

2 - Plantes sans étamines

1 - Plants with aborted stamens

2 - Plants without stamen

l'ensemble des hybrides de ce type de croisement, la fréquence dans les différentes classes de stérilité. Aucune plante n'a une stérilité pollinique inférieure à 10 et l'ensemble de l'histogramme présente une bonne symétrie. Deux classes supplémentaires de plantes sont également portées sur cet histogramme : la $1^{\text {re }}$ comprenant 2 plantes à étamines atrophiées et la $2^{\text {e }} 9$ plantes sans étamine. Nos observations polliniques sont assez semblables à celles de ROY (1978) qui a noté dans la descendance d'un croisement similaire, réalisé pour l'introduction de la résistance à la hernie et au Sclerotinia, une large variabilité de la fertilité en F2 (autofécondation des F1).

Ce croisement nous paraît beaucoup plus exploitable que les précédents car le grand nombre de descendants obtenus en G2 favorise une bonne expression de la variabilité et nous permettra de réaliser un tri plus efficace sur la valeur agronomique des géniteurs futurs.

\section{CONCLUSIONS ET PERSPECTIVES}

La culture in vitro d'embryons immatures permet d'augmenter le nombre d'hybrides obtenus. Cette technique peut être encore améliorée, principalement dans la $2^{\mathrm{e}}$ phase, lors des changements de concentration en sucre et au moment du repiquage en milieu non gélosé.

Les comportements méiotiques des hybrides observés ne sont pas en contradiction avec les hypothèses d'homéologie (U, 1935), seules quelques particularités peuvent être dues au choix des géniteurs. L'appariement entre les génomes différents observés principalement dans les croisements entre $B$. carinata et $B$. napus peut favoriser les recombinaisons, ce qui est souhaitable.

Il est regrettable que nous n'ayons pas pu observer le comportement méiotique des hybrides entre $B$. nigra et $B$. napus car HEYN (1977) avait observé une forte proportion de gamètes non réduits à la suite de ce croisement.

L'objectif principal de notre travail est l'obtention d'un système fiable d'androstérilité génocytoplasmique par création de lignées alloplasmiques. Du fait du nombre important de plantes obtenues en $2^{\mathrm{e}}$ génération et des familles qu'elles constituent en G3, G4..., il était indispensable de déterminer les études prioritaires. Ainsi avons-nous privilégié en G2, G3 et G4 les études concernant l'androstérilité et la restauration de la fertilité ; chaque plante ne subira pas un contrôle cytogénétique systématique mais un sondage sera réalisé sur l'ensemble des descendants. Nous ne pouvons donc pas mettre en évidence les éventuelles liaisons entre l'androstérilité et la présence d'aberrations chromosomiques. Ceci n'est pas le but de l'étude puisque nous cherchons, au contraire, à obtenir des systèmes d'androstérilité à déterminisme nucléo-cytoplasmique.

Pour éliminer les stérilités mâles dues à des aberrations chromosomiques, l'analyse cytogénétique est indispensable ; elle sera effectuée de façon systématique en $8^{\mathfrak{e}}$ ou $9^{\mathrm{e}}$ génération.

Ce choix de ne réaliser qu'un sondage au lieu d'une analyse systématique du comportement méiotique entre la $2^{\mathrm{e}}$ et la $8^{\mathrm{c}}$ génération permettra de consacrer plus de temps à l'étude de la stérilité mâle et de la restauration des diverses familles et d'en conserver un plus grand nombre.

L'obtention d'un système d'androstérilité cytoplasmique nécessite 3 types de géniteurs (mâle, stérile, mainteneur et restaurateur). Les résultats concernant la fertilité et la stérilité pollinique en $\mathrm{G} 2$ nous permettent de croire à l'obtention d'un tel système. D'après l'analyse bibliographique concernant ce type d'androstérilité, en particulier dans le cas du blé et du tournesol, le rétrocroisement systématique apparaît comme une méthode rapide et efficace pour l'obtention des familles androstériles, les mainteneurs ayant été trouvés par prospection dans l'espèce. Par contre, le rétrocroisement strict limite les possibilités d'obtenir des lignées à restauration efficace, cette méthode réduisant les recombinaisons. La lignée restauratrice de fertilité de la famille androstérile issue du croisement interspécifique $A \times B$ possède le cytoplasme de l'espèce $A$ et la presque totalité du génome de l'espèce $B$, une fraction de ce génome étant recombiné avec celui de $\mathrm{A}$. Le système de sélection que nous proposons repose donc sur le principe suivant : les plantes ayant l'indice de fertilité le plus élevé sont les plus aptes à la restauration. Il faut donc alterner les générations de rétrocroisement avec le colza et les générations défavorisant moins la recombinaison. Ainsi nous proposons le cycle suivant :

- en $2^{e}$ génération, les plantes les plus fertiles sont rétrocroisées par le colza.

- la $3^{\mathrm{e}}$ génération permet de trier les plantes les plus fertiles et de les autoféconder.

- en $4^{c}$ génération, l'aptitude à la restauration de la fertilité est jugée par croisement sur les familles stériles et les plantes sont intercroisées pour obtenir la génération suivante.

Le cycle reprend l'année suivante avec le rétrocroisement par le colza. Le test de fertilité est cependant remplacé par une analyse de l'aptitude à la restauration à chaque génération. Le test de l'aptitude à la restauration a l'avantage d'être plus près de la réalité mais son résultat est décalé d'un an. Ce système doit nous permettre de réaliser une sélection combinée du mâle-stérile et du restaurateur. 


\section{RÉFÉRENCES BIBLIOGRAPHIQUES}

Auriau P., Pluchard P., de Buyser J., 1973. Hérédité de la restauration de la fertilité mâle chez les blés hybrides. I. Résultats préliminaires. Ann. Amélior. Plantes, 23, 315-332.

Auriau P., Pluchard P., Chapuset T., Tallon P., 1979. Quel est l'avenir des blés hybrides? Ann. Amélior. Plantes, 29, 131-144.

Bannerot H., Boulidard L., Cauderon Y., Tempe J., 1974. Cytoplasmic male sterility transfer from Raphanus to Brassica. Proc. Eucarpia Meeting Cruciferae Vegetable. Crops Sect., 25-27th Sept. 1974, $52-54$.

Dosba F., Doussinault G., 1978. Création de lignées de blé présentant les caractéristiques agronomiques favorables d'Aegilops ventricosa. Ann. Amélior. Plantes, 28, 27-44.

Dickson M. H., 1975. G 1117 A, G 1102 A and G 1106 A cytosterile brocoli inbreds. Hortscience, 10, 535.

Edwardson J. R., 1970. Cytoplasmic male sterility. Bot. Rev., 36, 341-420.

Heyn F. W., 1977. Analysis of unreduced gametes in the Brassiceae by the crosses with species and ploidy levels. $Z$. Pflanzenzücht, 78 , 13-30.

Howard H. W., 1942. Self incompatibility in polyploid forms of Brassica and Raphanus. Nature, 149, 302-303.

Iwasa S., 1951. Studies on artificial triploid and hexaploid Brassica types having the three genomes ABC (in Japanese). Sci. Bull. Fac. Agric. Kyushu Univ., 13, $90-99$ (in Plant Breed. Abstr., 24, 1202). Kihara H., 1951. Substitution of nucleus and its effects on genome manifestations. Cytologia, 16, 177-198.

Leclercq P., 1969. Une stérilité mâle cytoplasmique chez le tournesol. Ann. Amélior. Plantes, 19, 99-106.

Leclercq P., 1971. La stérilité mâle cytoplasmique du tournesol. I. Premières études sur la restauration de la fertilité. Ann. Amélior. Plantes, 21, 45-54.

Mizushima U., 1944. Studies of some auto and allopolyploids made in Brassica, Eruca and Raphanus. Agric. and Hort. Tokyo, 19, 743774 (Abstr. in Heredity, 4, 399).

Mizushima U., 1950. On several artificial allopolyploids obtained in the tribe Brassicae of Cruciferae. Tohoku J. Agric. Res., 1, 15-27.

Monnier M., 1974. Culture in vitro de l'embryon immature de Capsella bursa pastoris Moench. Thèse d'état - Université de Paris VI, $186 \mathrm{p}$.
Morice J., 1963. Les relations entre les espèces cultivées du genre Brassica et les possibilités d'amélioration du colza au moyen de croisements interspécifiques. Ann. Amélior. Plantes, 13, 221-252.

Morinaga T., 1929. Interspecific hybridization in Brassica. I. The cytology of F1 hybrids of Brassica napella and various other species with 10 chromosomes. Cytologia, 1, 16-27.

Morinaga T., 1931. Interspecific hybridization in Brassica. IV. The cytology of F1 hybrids of Brassica carinata and some other species with 10 chromosomes. Cytologia, 3, 77-83.

Ogura H., 1968. Studies of the new male-sterility in Japanese radish, with special reference to the utilization of this sterility towards the practical raising of hybrid seeds. Mem. Fac. Agric. Kagoshima Univers., 6, 39-78.

Pearson O. H., 1972. Cytoplasmically inherited male sterility characters and flavor components from the species cross Brassica nigra $\times$ Brassica oleracea. J. Amer. Soc. Hort. Sci., 97, 397-402.

Rousselle P., 1981. Etude de systèmes d'androstérilité chez le colza (Brassica napus). Thèse Doctorat d'Ingénieur, ENSA Rennes, 812, $109 \mathrm{p}$.

Roy N. N., 1978. A study on disease variation in the populations of an interspecific cross of Brassica juncea $\times$ Brassica napus. Euphytica, 27, 145-149.

Shiga T., 1970. Rape breeding by interspecific crossing between Brassica napus and Brassica campestris in Japan. Jap. Agric. Res. Quaterly, 5, 5-10.

Shiga T., 1976. Studies on heterosis breeding using cytoplasmic male sterility in rapeseed Brassica napus L. Bull. Nat. Inst. Agric. Sci. Tokyo, Série D 27, 1-101.

Sinskala E. N., 1927. Geno-systematical investigations of cultivated Brassica. Bull. appl. Bot. Genet. Pl. Breed., 17, 3-166.

Thompson K. F., 1972. Cytoplasmic male sterility in oil-seed rape. Heredity, 29, 253-257.

Tsunewaki K., 1978. Genetic effects of 25 alien cytoplasma on plant height and its component parts in common wheat. Wheat Inf. Serv., 47-48, 56-58.

U N., 1935. Genome analysis in Brassica with special reference to the experimental formation of Brassica napus and peculiar mode of fertilization. Jap. J. Bot., 7, 389-452.

Wilson J. A., Ross W. M., 1962. Male sterility interaction of the Triticum aestivum nucleus and Triticum timopheevi cytoplasm. Wheat Inf. Ser., 14, 29. 